\title{
Identification of the molecular mechanisms underlying dilated cardiomyopathy via bioinformatic analysis of gene expression profiles
}

\author{
HU ZHANG ${ }^{1}$, ZHUO YU ${ }^{2}$, JIANCHAO HE ${ }^{1}$, BAOTONG HUA $^{2}$ and GUIMING ZHANG ${ }^{1}$ \\ Departments of ${ }^{1}$ Cardiaovascular Surgery and ${ }^{2}$ Cardiology, The First Affiliated Hospital of Kunming Medical University, \\ Kunming, Yunnan 650032, P.R. China
}

Received March 26, 2015; Accepted April 21, 2016

DOI: 10.3892/etm.2016.3953

\begin{abstract}
In the present study, gene expression profiles of patients with dilated cardiomyopathy (DCM) were re-analyzed with bioinformatics tools to investigate the molecular mechanisms underlying DCM. Gene expression dataset GSE3585 was downloaded from Gene Expression Omnibus, which included seven heart biopsy samples obtained from patients with DCM and five healthy controls. Differential analysis was performed using a Limma package in $\mathrm{R}$ to screen for differentially expressed genes (DEGs). Functional enrichment analysis was subsequently conducted for DEGs using the Database for Annotation, Visualization and Integration Discovery. A protein-protein interaction (PPI) network was constructed using information from Search Tool for the Retrieval of Interacting Genes software. A total of 89 DEGs were identified in the patients with DCM, including 67 upregulated and 22 downregulated genes. Functional enrichment analysis demonstrated that the downregulated genes predominantly encoded chromosomal proteins and transport-related proteins, which were significantly associated with the biological processes of 'nucleosome assembly', 'chromatin assembly', 'protein-DNA complex assembly', 'nucleosome organization' and 'DNA packaging' (H1 histone family member 0, histone cluster $1 \mathrm{H} 1 \mathrm{c}$, histone cluster $1 \mathrm{H} 2 \mathrm{bd}$ and $\mathrm{H} 2 \mathrm{~A}$ histone family member Z). The upregulated genes detected in the present study encoded secreted proteins or phosphotransferase, which were associated with biological processes including "cell adhesion' [connective tissue growth factor (CTGF)], 'skeletal system development' [CTGF and insulin-like growth factor
\end{abstract}

Correspondence to: Dr Guiming Zhang, Department of Cardiaovascular Surgery, The First Affiliated Hospital of Kunming Medical University, 295 Xichang Road, Kunming, Yunnan 650032, P.R. China

E-mail: zhexming@163.com

Key words: dilated cardiomyopathy, gene expression profile, differentially expressed genes, functional enrichment analysis, protein-protein interaction network binding protein 3 (IGFBP3)], 'muscle organ development' (SMAD7) and 'regulation of cell migration' [SMAD7, IGFBP3 and insulin receptor (INSR)]. Notably, signal transducer and activator of transcription 3, SMAD7, INSR, CTGF, exportin 1, IGFBP3 and phosphatidylinositol-4,5-bisphosphate 3-kinase, catalytic subunit alpha were hub nodes with the higher degree in the PPI network. Therefore, the results of the present study suggested that DEGs may alter the biological processes of 'nucleosome formation', 'cell adhesion', 'skeletal system development', 'muscle organ development' and 'regulation of cell migration' in the development of DCM.

\section{Introduction}

Dilated cardiomyopathy (DCM) is the most frequent type of non-ischemic cardiomyopathy worldwide, with an estimated prevalence of 1 in 2,500 people and an incidence of $7 / 100,000$ people annually $(1,2)$. DCM is characterized by dilatation and reduced contractile function of the left and right ventricles, which may lead to progressive heart failure and sudden cardiac-associated mortality in $4.5-79.3 \%$ of sufferers at 3 years (3). Therefore, the early diagnosis and treatment of DCM is important in order to prevent a poor prognosis.

The molecular mechanisms underlying DCM have been extensively explored in an attempt to provide effective diagnostic and therapeutic methods for DCM. These previous studies have demonstrated that DCM is associated with mutations in genes encoding cytoskeletal, contractile or inner-nuclear membrane proteins $(4,5)$, including actin, desmin (6) and lamins A and C (7). Furthermore, it has been demonstrated that mutations in sarcomere protein genes account for $\sim 10 \%$ of cases of familial DCM $(8,9)$. Mitochondrial DNA mutations and mitochondrial abnormalities have also been implicated in the pathogenesis of DCM by altering myocardial ATP generation (10), including mitochondrial Hsp40 which has a crucial role in preventing DCM (11). As a result, various potential biomarkers have been elucidated, including high-sensitivity cardiac troponin $\mathrm{T}$ (12), serum matrix metalloproteinase-3 (13) and serum Tenascin-C (14). However, the mechanisms and pathogenesis underlying DCM remain poorly understood.

Microarray technology enables the measurement of global gene expression levels, and thus facilitates the identification of 
A

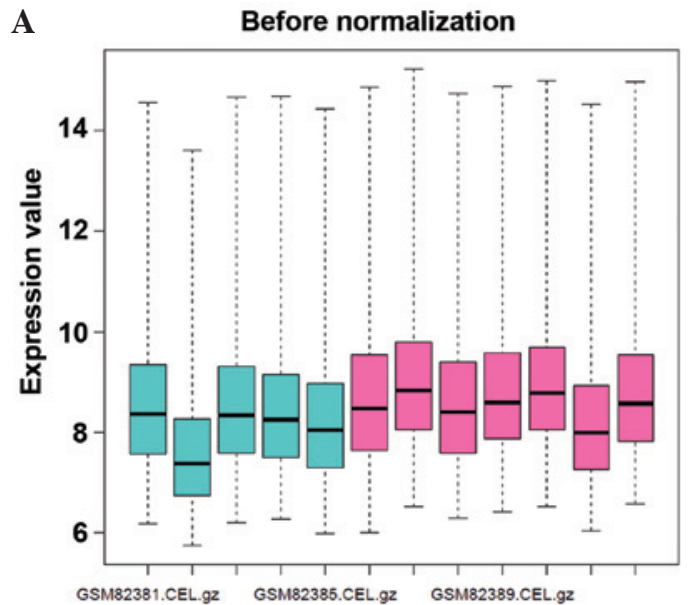

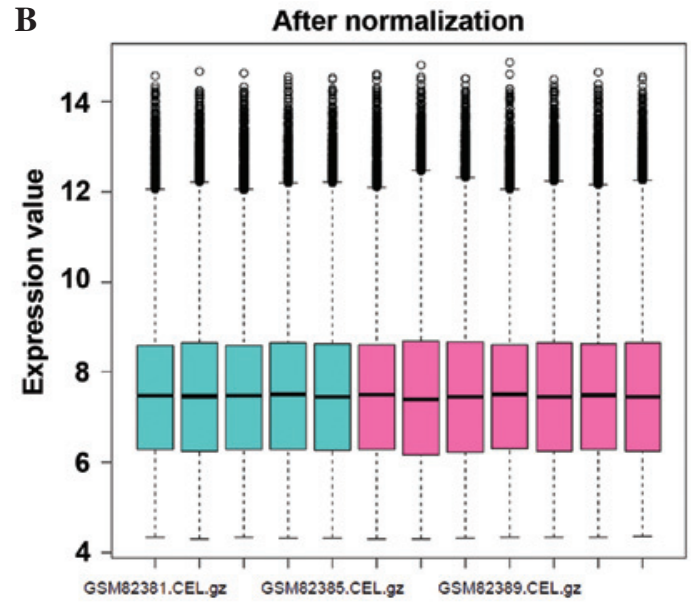

Figure 1. Box plots of gene expression data (A) before and (B) after normalization. Dilated cardiomyopathy samples are presented in blue, whereas normal controls are pink. Medians are represented as black horizontal lines in the boxes.

crucial genes and altered biological processes in DCM (15-17). Although Barth et al (16) have previously identified a common gene expression signature in DCM via microarray data, deeper analysis of the massive gene expression dataset is required via bioinformatics. Therefore, the present study reanalyzed gene expression data (16) to identify differentially expressed genes (DEGs) which may contribute to the development of DCM. Following this, functional enrichment and protein-protein interaction (PPI) analyses of the DEGs were performed using various bioinformatics tools. These findings may advance understanding of the molecular mechanisms underlying DCM.

\section{Materials and methods}

Gene expression data. Gene expression dataset (accession number, GSE3585) (16) was downloaded from Gene Expression Omnibus (http://ncbi.nlm.nih.gov/geo/). The GSE3585 dataset included seven heart biopsy samples obtained from patients with DCM at time of transplantation (GSM82386-GSM82392) and five non-failing (NF) heart biopsies from NF donor hearts (GSM82381-GSM82386). Gene expression levels were measured using Affymetrix Human Genome U133A Array (Affymetrix Inc., Santa Clara, CA, USA).

Data pre-processing and differential analysis. Raw data (CEL file) were read by an Affy package (http://bioconductor.org/packages/release/bioc/html/affy.html) (18) of $R$ (version 3.1.2; http://r-project.org/) (19). Probes detected in $>50 \%$ of samples were retained. Background correction, data normalization and determination of expression levels was conducted using a Robust Multi-array Average analysis method (20).

Differential analysis was performed using the linear model of the lmFit and empirical Bayes moderated t test provided by the Limma package (http://bioconductor.org/packages/release/bioc/html/limma.html) (21). Ilog Fold Change (FC) $1>0.3$ and false discovery rate $($ FDR) $<0.05$ were set as the cut-offs to screen DEGs between DCM and NF hearts.

Functional enrichment analysis. Functional enrichment analysis of the DEGs was conducted using the Database for Annotation, Visualization and Integration Discovery (version 6.7; http://david.abcc.ncifcrf.gov/) (22), with a two-tailed Fisher exact test based on the hypergeometric distribution. Benjamini corrected P-values of $<0.05$ were set as the cut-off to screen out significant Gene Ontology biological process (GOBP) terms (23) and Kyoto Encyclopedia of Genes and Genomes (KEGG) pathways (24).

Construction of PPInetwork. A PPI network was constructed for the DEGs using the Search Tool for the Retrieval of Interacting Genes (STRING; version 9.1; http://string-db.org/) (25). Interacting pairs with a combined score $>0.4$ were selected for the construction of the PPI network. Proteins serve as the 'nodes' in the network and each pairwise protein interaction is represented by an undirected link. Degree was calculated for each node, which corresponds to the number of interactions of a protein with other proteins. Hub genes were subsequently selected according to the degree.

\section{Results}

Microarray data analysis identified DEGs between the DCM and control groups. Following data normalization, the mean expression levels of the gene expression profiles were presented in one consistent line (Fig. 1). Subsequently, according to the criteria of $\log \mathrm{FCl}>0.3$ and $\mathrm{FDR}<0.05,89$ DEGs were detected between the DCM and NF hearts, including 22 downregulated and 67 upregulated genes. A greater number of upregulated genes were detected, as compared with the downregulated genes, which demonstrated that the DEGs had a tendency to upregulate in DCM. Based on the DEGs, the DCM samples were predominantly separated from the NF controls, implying the reliability of the DEGs (Fig. 2).

$G O$ and KEGG pathway analysis revealed the functions of $D E G$ s involved in DCM. Functional enrichment analysis was performed for the downregulated and upregulated genes, respectively. For the downregulated genes, two clusters were obtained using the Protein Information Resource. The first cluster was demonstrated to be associated with 'chromosomal proteins', including $\mathrm{H} 1$ histone family member 0 


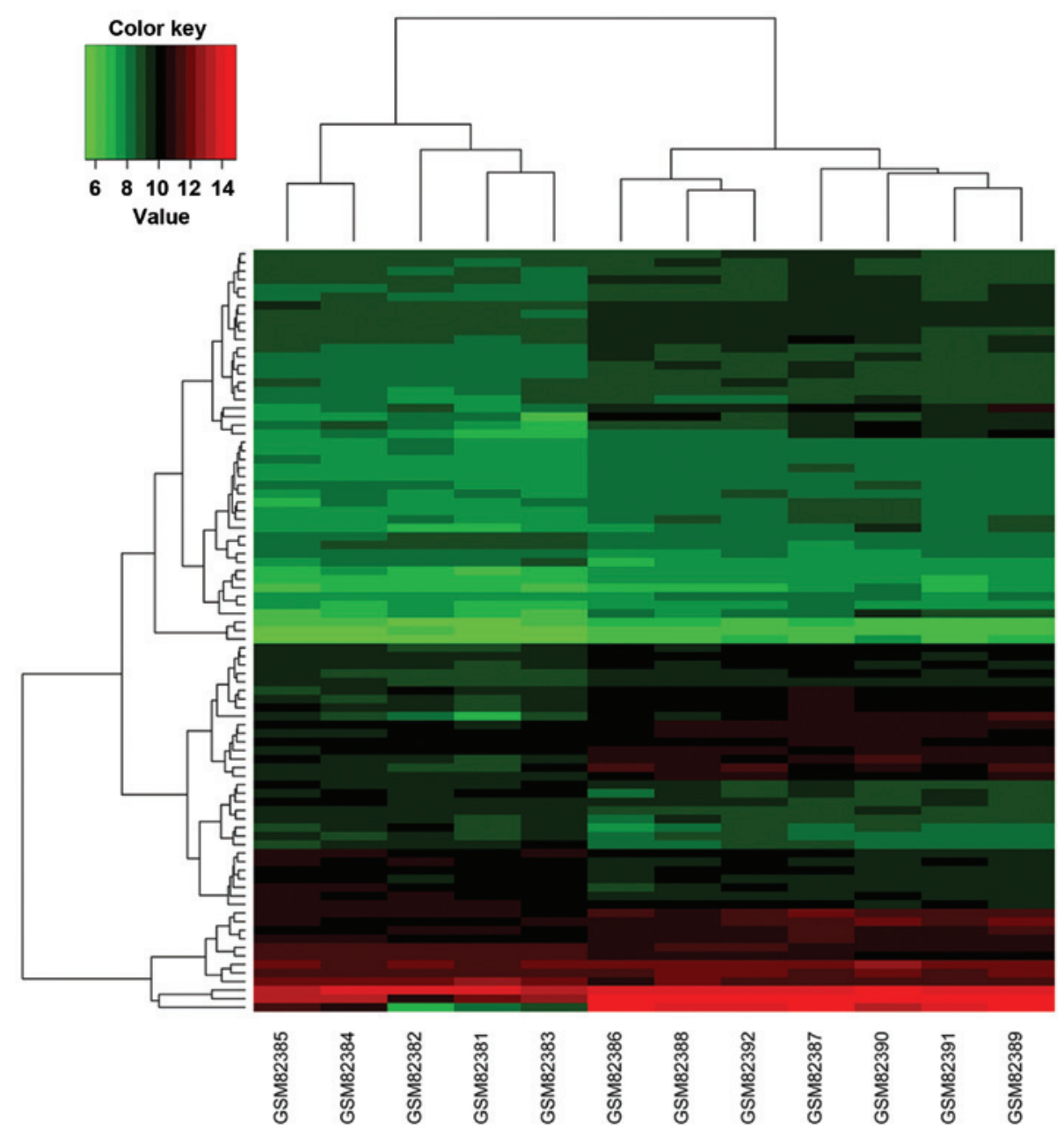

Figure 2. Heat map of hierarchical clustering analysis of the differentially expressed genes.

$(\mathrm{H} 1 \mathrm{~F} 0 ; \log \mathrm{FC}=-0.61 ; \mathrm{FDR}=1.91 \mathrm{E}-02), \mathrm{H} 2 \mathrm{~A}$ histone family member $\mathrm{Z}$ (H2AFZ; logFC=-0.47; FDR=1.94E-02), histone cluster $1 \mathrm{H} 1 \mathrm{c}$ (HIST1H1C; $\log \mathrm{FC}=-0.71$; FDR=3.69E-02) and histone cluster $1 \mathrm{H} 2 \mathrm{bd}$ (HIST1H2BD; $\log \mathrm{FC}=-0.69$; $\mathrm{FDR}=2.89 \mathrm{E}-02$ ). The second cluster was associated with transmembrane transport-related proteins, including STEAP family member $3(\log F C=-0.92 ; F D R=4.39 E-02)$, potassium voltage-gated channel subfamily D member $3(\log \mathrm{FC}=-0.54$; $\mathrm{FDR}=4.48 \mathrm{E}-02)$ and insulin-like growth factor 2 receptor $(\log \mathrm{FC}=-0.44 ; \mathrm{FDR}=4.76 \mathrm{E}-02)$. Furthermore, downregulated genes (H1F0, HIST1H2BD, HIST1H1C and H2AFZ) were significantly enriched in the biological processes of 'nucleosome assembly' ( $\mathrm{P}=1.22 \mathrm{E}-04)$, 'chromatin assembly' ( $\mathrm{P}=1.35 \mathrm{E}-04)$, 'protein-DNA complex assembly' $(\mathrm{P}=1.55 \mathrm{E}-04)$, 'nucleosome organization' $(\mathrm{P}=1.65 \mathrm{E}-04)$ and 'DNA packaging' $(\mathrm{P}=3.25 \mathrm{E}-04$; Table $\mathrm{I})$.

For the upregulated genes, two clusters were also acquired using the Protein Information Resource, including 'secreted proteins' such as connective tissue growth factor (CTGF; $\log \mathrm{FC}=0.84 ; \mathrm{FDR}=3.04 \mathrm{E}-02$ ) and insulin-like growth factor binding protein 3 (IGFBP3; $\log \mathrm{FC}=0.84 ; \mathrm{FDR}=3.04 \mathrm{E}-02)$ in cluster 1, and 'phosphotransferase' such as phosphatidylinositol-4,5-bisphosphate 3-kinase, catalytic subunit alpha (PIK3CA; $\log F C=0.59 ; \mathrm{FDR}=4.44 \mathrm{E}-02$ ) and insulin receptor (INSR; $\log \mathrm{FC}=0.61 ; \mathrm{FDR}=2.27 \mathrm{E}-02$ ) in cluster 2 . Additionally, six GOBP terms were detected in the upregulated genes $(\mathrm{P}<0.05)$, including 'cell adhesion' (CTGF), 'skeletal system development' (CTGF and IGFBP3), 'muscle organ development' (SMAD7; $\log F C=0.56 ; \mathrm{FDR}=4.39 \mathrm{E}-02$ ), and 'regulation of cell migration' (SMAD7, IGFBP3 and INSR) (Table II). No significant pathways were enriched for the DEGs.

PPI network analysis identifies crucial genes for DCM. A PPI network containing 22 DEGs was constructed using the protein interaction information obtained from STRING (Fig. 3). By calculating the degree of each gene in the network, seven DEGs were considered to be crucial for DCM with a degree of $>3$, including signal transducer and activator of transcription 3 (STAT3; degree, 7; $\log \mathrm{FC}=-0.86$; FDR= 2.27E-02), SMAD7 (degree, 6), INSR (degree, 5), exportin 1 (XPO1; degree, 5; $\log \mathrm{FC}=0.62 ; \mathrm{FDR}=2.74 \mathrm{E}-02$ ), CTGF (degree, 4), IGFBP3 (degree, 4) and PIK3CA (degree, 4).

\section{Discussion}

In the present study, by reanalyzing the gene expression profiles of DCM and NF samples (16), 89 DEGs were identified in DCM patients, including 22 downregulated and 67 upregulated genes. Downregulated genes were demonstrated to encode chromosomal proteins and transmembrane 
Table I. Significantly enriched functional terms of downregulated genes.

\begin{tabular}{|c|c|c|c|c|}
\hline Category & Enrichment score & Terms & P-value & Genes \\
\hline \multicolumn{5}{|l|}{ SP_PIR_KEYWORDS } \\
\hline Annotation Cluster 1 & 1.689911619479253 & Chromosomal protein & $3.72 \mathrm{E}-04$ & $\begin{array}{l}\text { H1F0, HIST1H2BD, } \\
\text { HIST1H1C, H2AFZ }\end{array}$ \\
\hline Annotation Cluster 2 & 0.32418860902901725 & Transport & $2.25 \mathrm{E}-01$ & $\begin{array}{l}\text { STEAP3, KCND3, } \\
\text { IGF2R, SELENBP1 }\end{array}$ \\
\hline \multicolumn{5}{|l|}{ GO_BP_FAT } \\
\hline Annotation Cluster 1 & 2.7555946833424456 & & & \\
\hline GO:0006334 & & Nucleosome assembly & $1.22 \mathrm{E}-04$ & $\begin{array}{l}\text { H1F0, HIST1H2BD, } \\
\text { HIST1H1C, H2AFZ }\end{array}$ \\
\hline GO:0031497 & & Chromatin assembly & $1.35 \mathrm{E}-04$ & $\begin{array}{l}\text { H1F0, HIST1H2BD, } \\
\text { HIST1H1C, H2AFZ }\end{array}$ \\
\hline GO:0065004 & & Protein-DNA complex assembly & $1.55 \mathrm{E}-04$ & $\begin{array}{l}\text { H1F0, HIST1H2BD, } \\
\text { HIST1H1C, H2AFZ }\end{array}$ \\
\hline GO:0034728 & & Nucleosome organization & $1.65 \mathrm{E}-04$ & $\begin{array}{l}\text { H1F0, HIST1H2BD, } \\
\text { HIST1H1C, H2AFZ }\end{array}$ \\
\hline GO:0006323 & & DNA packaging & $3.25 \mathrm{E}-04$ & $\begin{array}{l}\text { H1F0, HIST1H2BD, } \\
\text { HIST1H1C, H2AFZ }\end{array}$ \\
\hline
\end{tabular}

The functional enrichment analysis was performed using the Database for Annotation, Visualization and Integration Discovery. Gene symbols are listed in accordance with the National Center for Biotechnology Information database. SP, swiss-prot; PIR, protein information resource; GO, gene ontology; BP, biological process; FAT, functional annotation tool; H1F0, H1 histone family member 0; HIST1H2BD, histone cluster 1 H2bd; HIST1H1C, histone cluster $1 \mathrm{H} 1 \mathrm{c}$; H2AFZ, H2A histone family member Z; STEAP3, STEAP family member 3; KCND3, potassium channel voltage gated shal related subfamily D member 3; IGFR2, insulin-like growth factor 2 receptor; SELENBP1, selenium binding protein 1.

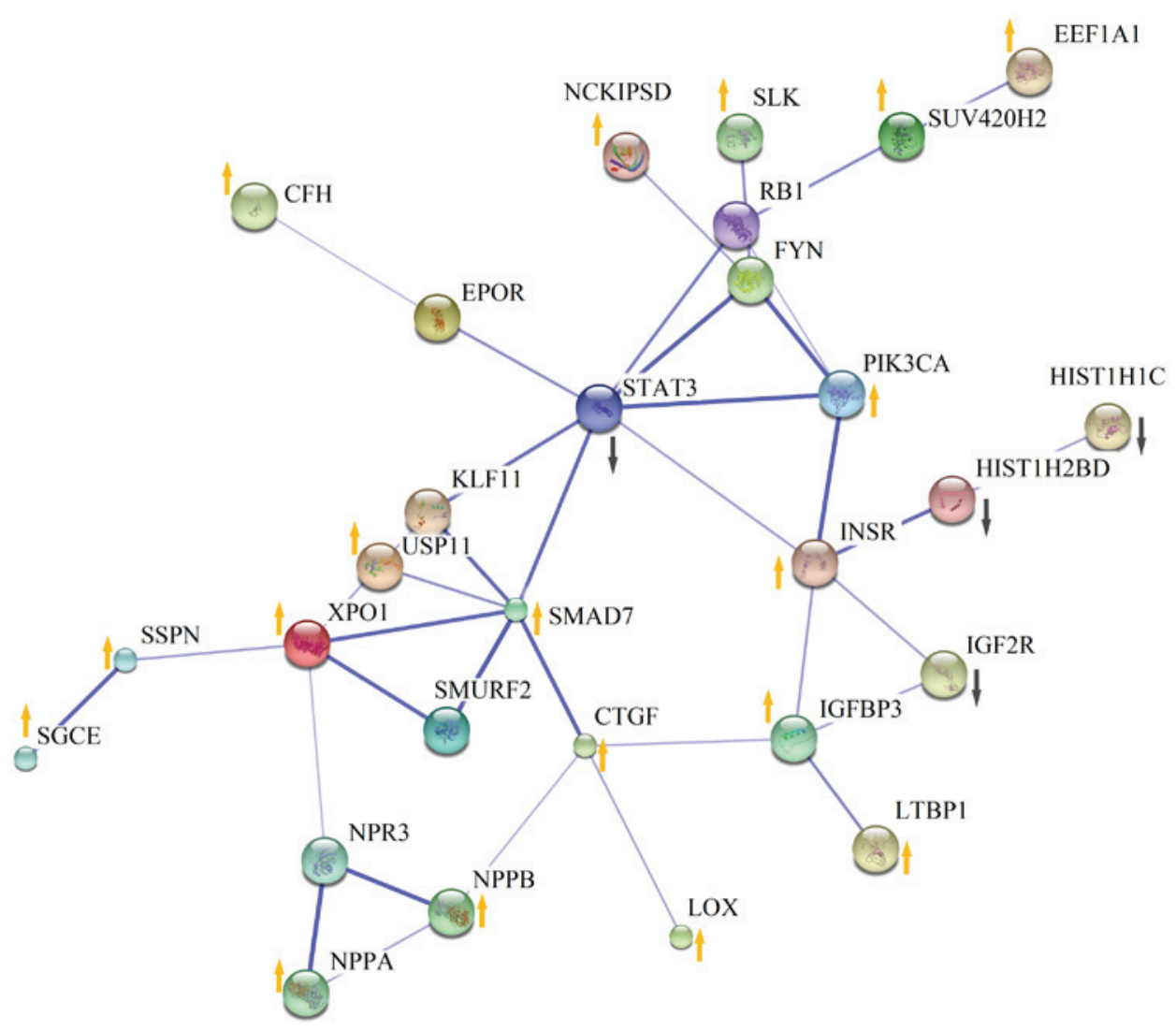

Figure 3. Protein-protein interaction network for the differentially expressed genes. Each node represents the protein and each line represents the interaction between paired proteins. 
Table II. Significantly enriched functional terms of upregulated genes.

\begin{tabular}{lclll}
\hline Category & Enrichment score & \multicolumn{1}{c}{ Terms } & P-value & \multicolumn{1}{c}{ Genes } \\
\hline $\begin{array}{c}\text { SP_PIR_KEYWORDS } \\
\text { Annotation cluster 1 }\end{array}$ & 1.1489102585231292 & Secreted & & \\
& & & $9.05 E-03$ & AEBP1, LTBP1, SPOCK1 \\
& & & FRZB, OMD, CTGF, CFH, \\
Annotation cluster 2 & 1.1141850825108304 & Phosphotransferase & 2.94E-02 & LAMB1, IGFBP3, NPPA \\
& & & & ROR1, PIK3CA, CLK1, \\
& & &
\end{tabular}

\section{GOTERM_BP_FAT}

\section{Annotation cluster 1}

GO:0007155

2.187528735182074

Cell adhesion

$6.23 \mathrm{E}-03$

OMD, AEBP1, CTGF, PKD2, SPOCK1, SGCE, DLG5, LAMB1, SSPN

\section{Annotation cluster $2 \quad 1.9708171852370016$ GO:0001501}

Annotation cluster 3 GO:0007517

\section{Annotation cluster 4 GO:0030334}

\section{Annotation cluster 5 GO:0007507}

\author{
Annotation cluster 6 \\ GO:0006163
}

1.5566908357862366

1.3188719375499292

1. 2806966535392923

Heart development

1.1685982822293663

Purine nucleotide metabolic process

Muscle organ development

Regulation of cell migration
Skeletal development

8.69E-03

9.90E-03

4.57E-03

SMAD7, LAMB1, IGFBP3, INSR, TPM1

$1.06 \mathrm{E}-02$

SMAD7, PKD2, INSR, TPM1, MYH10

MGEA5, ATP10D, ATP13A3, NPPA

Functional enrichment analysis was performed using the Database for Annotation, Visualization and Integration Discovery. Only the highest significantly enriched term for each annotation cluster are presented. Gene symbols are listed in accordance with the National Center for Biotechnology Information database. SP, swiss-prot; PIR, protein information resource; GO, gene ontology; BP, biological process .

transport-related proteins, which were associated with 'nucleosome assembly', 'chromatin assembly', 'protein-DNA complex assembly', 'nucleosome organization' and 'DNA packaging'. Upregulated genes were enriched into two annotation clusters, 'secreted proteins' and 'phosphotransferase', which were associated with 'cell adhesion', 'skeletal system development', 'muscle organ development' and 'regulation of cell migration'. Notably, upregulated SMAD7, STAT3, INSR, XPOI, CTGF, $I G F B P 3$ and PIK3CA were hub nodes in the PPI network.

Several histone family members were demonstrated to be downregulated in DCM, including $H 1 F O, H 2 A F Z$, HISTIH2BD and HISTIHIC. To the best of our knowledge, this is the first report of the association between these genes and DCM. Histones are basic nuclear proteins that are responsible for the nucleosome structure of chromosomal fiber in eukaryotes (26). Previous studies have indicated that histone acetylation/deacetylation regulates cardiac morphogenesis, growth and contractility $(27,28)$. The present results demonstrated that these downregulated histone family member genes were significantly enriched in GO terms including 'nucleosome assembly', 'chromatin assembly', 'protein-DNA complex assembly', 'nucleosome organization' and 'DNA packaging'. Therefore, the downregulation of these genes may contribute to the blockade of nucleosome formation. As previously described, the untranscribed regions of eukaryotic genomes are packaged into nucleosomes, which may repress gene expression in general (29). Depletion of nucleosomal histones may lead to the silencing of specific genes (30). Therefore, we hypothesize that the downregulation of histones may induce the overexpression of numerous genes associated with the development of DCM via nucleosome formation, which may explain the tendency for upregulation of DEGs observed in the present study.

In the present study, 67 upregulated genes were detected in DCM. CTGF, which is a profibrotic cytokine, was demonstrated to be associated with 'cell adhesion' and 'skeletal system development'. Previous studies have indicated that CTGF has a key role in the deleterious process of cardiac fibrosis, which is a hallmark of DCM $(31,32)$. Furthermore, it has been demonstrated that CTGF is highly induced in viral myocarditis and its silencing may counteract cardiac fibrosis and heart muscle 
dysfunction (33). CTGF was also demonstrated to be consistently upregulated in DCM patients in the present study, which may contribute to abnormal cardiac fibrosis. Moreover, it has been demonstrated that IGFBP3, which is a member of the insulin-like growth factor binding protein family, is upregulated in the failing hearts of DCM patients $(34,35)$. Hassfeld et al $(36)$ regard IGFBP3 as an independent predictors of a poor prognosis in patients with DCM. Notably, the results of the present study indicated that upregulated IGFBP3 was related to 'skeletal system development' and 'regulation of cell migration'. Similarly, INSR was upregulated in DCM and was associated with 'regulation of cell migration'. The binding of insulin to INSR stimulates glucose uptake which affects muscle function in vitro (37), thus the upregulated INSR may be associated with muscle contractility. Furthermore, SMAD7 was upregulated in DCM patients, which is one feature of dysregulated extracellular matrix degradation which may lead to cardiac fibrosis in patients with diabetic cardiomyopathy (38). DCM is characterized by abnormal contractile function $(39,40)$. The DEGs mentioned above (CTGF, SMAD7, IGFBP3 and INSR) were found to be enriched in 'cell adhesion', 'skeletal system development', 'muscle organ development' and 'regulation of cell migration', and abnormalities in these processes may contribute to weakened contractile function. Notably, CTGF, SMAD7, IGFBP3 and INSR were also demonstrated to be hub nodes in the PPI network, suggesting their predominant roles among the DEGs. These four DEGs may be future therapeutic targets for the treatment of DCM.

Although the STAT3, XPO1 and PIK3CA GO terms were not enriched in the present study, they did form hub nodes in the PPI network, thus suggesting that these genes are also important in DCM. This is supported by previous studies. Podewski et al (41) observed that the protein expression levels of STAT3 were significantly decreased in the cardiomyocytes of patients with end-stage DCM. Furthermore, using the knockout technique, Hilfiker-Kleiner et al (42) demonstrated reduced myocardial capillary density and increased interstitial fibrosis in STAT3-deficient mice, leading to DCM with impaired cardiac function and premature mortality. PIK3CA, a stress-associated lipid kinase, has been shown to be important for maintaining cardiac structure and function, and its upregulation ultimately prolonged the survival of a mouse model of DCM $(43,44)$. In addition, XPO1 has been reported to be a key nucleocytoplasmic transport-related gene in DCM $(45,46)$, and upregulation of XPO1 may be involved in DCM by mediating the nuclear export of several molecules that regulate cardiac growth and development, including a member of the Rho family of GTPases, Ras homolog family member $\mathrm{U}(\mathrm{RhoU})$, and a transcriptional coactivator and cytoskeleton regulator, four and a half LIM domains 3 (FHL3) (46). It has been reported that the loss of RhoU results in the mis-localization of cell adhesion molecules, including Alcama and $\mathrm{N}$-cadherin, to the cytoplasm and the apical and basal cell membrane, instead of the atrioventricular cardiomyocyte cell junctions, resulting in failure to form the atrioventricular canal and loop the linear heart tube and thus influencing cardiac function (47). Furthermore, downregulated FHL3 expression has been associated with the systolic dysfunction of DCM patients by inhibiting the expression of myosin heavy chain isoform $2 \mathrm{a}(48,49)$.
In conclusion, the results of the present study suggested that downregulated histones may lead to the overexpression of DCM-specific genes via damage to the nucleosome. Furthermore, upregulated genes, including CTGF, SMAD7, IGFBP3 and INSR, may lead to weakened contractile function via various biological pathways associated with muscle development. STAT3, XPO1 and PIK3CA may also be important for maintaining heart function. There were some limitations to the results of the present study. The DEGs identified by microarray data were not validated using real-time polymerase chain reaction and there was no intervention or patient follow-up performed. Despite these limitations, the present findings may advance the understanding of the pathogenesis of DCM and provide novel targets for clinical treatment and diagnosis.

\section{Acknowledgements}

The present study was supported by the Natural Science Foundation of China (grant no. 31360227).

\section{References}

1. Hershberger RE, Hedges DJ and Morales A: Dilated cardiomyopathy: The complexity of a diverse genetic architecture. Nat Rev Cardiol 10: 531-547, 2013

2. Jefferies JL and Towbin JA: Dilated cardiomyopathy. Lancet 375 : 752-762, 2010.

3. Goldberger JJ, SubaÄ H, Patel T, Cunnane R and Kadish AH: Sudden cardiac death risk stratification in patients with nonischemic dilated cardiomyopathy. J Am Coll Cardiol 63: 1879-1889, 2014.

4. Gerull B, Gramlich M, Atherton J, McNabb M, Trombitás K, Sasse-Klaassen S, Seidman JG, Seidman C, Granzier H, Labeit S, et al: Mutations of TTN, encoding the giant muscle filament titin, cause familial dilated cardiomyopathy. Nat Genet 30: 201-204, 2002.

5. Villard E, Duboscq-Bidot L, Charron P, Benaiche A, Conraads V, Sylvius N and Komajda M: Mutation screening in dilated cardiomyopathy: Prominent role of the beta myosin heavy chain gene. Eur Heart J 26: 794-803, 2005.

6. Taylor MR, Slavov D, Ku L, Di Lenarda A, Sinagra G, Carniel E, Haubold K, Boucek MM, Ferguson D, Graw SL, et al: Prevalence of desmin mutations in dilated cardiomyopathy. Circulation 115: 1244-1251, 2007

7. Schmidt $\mathrm{HH}$ and Lochs $\mathrm{H}$ : Lamin A/C gene mutation associated with dilated cardiomyopathy with variable skeletal muscle involvement. Circulation 103: E20, 2001.

8. Frazier AH, Ramirez-Correa GA and Murphy AM: Molecular mechanisms of sarcomere dysfunction in dilated and hypertrophic cardiomyopathy. Prog Pediatr Cardiol 31: 29-33, 2011.

9. Kamisago M, Sharma SD, DePalma SR, Solomon S, Sharma P, McDonough B, Smoot L, Mullen MP, Woolf PK, Wigle ED, et al: Mutations in sarcomere protein genes as a cause of dilated cardiomyopathy. N Engl J Med 343: 1688-1696, 2000.

10. Arbustini E, Diegoli M, Fasani R, Grasso M, Morbini P, Banchieri N, Bellini O, Dal Bello B, Pilotto A, Magrini G, et al: Mitochondrial DNA mutations and mitochondrial abnormalities in dilated cardiomyopathy. Am J Pathol 153: 1501-1510, 1998

11. Hayashi M, Imanaka-Yoshida K, Yoshida T, Wood M, Fearns C, Tatake RJ and Lee JD: A crucial role of mitochondrial Hsp40 in preventing dilated cardiomyopathy. Nat Med 12: 128-132, 2006.

12. Kawahara C, Tsutamoto T, Nishiyama K, Yamaji M, Sakai H, Fujii M, Yamamoto $\mathrm{T}$ and Horie M: Prognostic role of high-sensitivity cardiac troponin T in patients with nonischemic dilated cardiomyopathy. Circ J 75: 656-661, 2011.

13. Ohtsuka T, Nishimura K, Kurata A, Ogimoto A, Okayama H and Higaki J: Serum matrix metalloproteinase-3 as a novel marker for risk stratification of patients with nonischemic dilated cardiomyopathy. J Card Fail 13: 752-758, 2007. 
14. Terasaki F, Okamoto H, Onishi K, Sato A, Shimomura H, Tsukada B, Imanaka-Yoshida K, Hiroe M, Yoshida T, Kitaura Y, et al: Higher serum tenascin-C levels reflect the severity of heart failure, left ventricular dysfunction and remodeling in patients with dilated cardiomyopathy. Circ J 71 327-330, 2007

15. Barrans JD, Allen PD, Stamatiou D, Dzau VJ and Liew CC: Global gene expression profiling of end-stage dilated cardiomyopathy using a human cardiovascular-based cDNA microarray. Am J Pathol 160: 2035-2043, 2002.

16. Barth AS, Kuner R, Buness A, Ruschhaupt M, Merk S, Zwermann L, Kääb S, Kreuzer E, Steinbeck G, Mansmann U, et al: Identification of a common gene expression signature in dilated cardiomyopathy across independent microarray studies. J Am Coll Cardiol 48: 1610-1617, 2006.

17. Camargo A and Azuaje F: Identification of dilated cardiomyopathy signature genes through gene expression and network data integration. Genomics 92: 404-413, 2008

18. Gautier L, Cope L, Bolstad BM and Irizarry RA: Affy-analysis of Affymetrix GeneChip data at the probe level. Bioinformatics 20: 307-315, 2004.

19. Gentleman RC, Carey VJ, Bates DM, Bolstad B, Dettling M, Dudoit S, Ellis B, Gautier L, Ge Y, Gentry J, et al: Bioconductor: Open software development for computational biology and bioinformatics. Genome Biol 5: R80, 2004

20. Irizarry RA, Hobbs B, Collin F, Beazer-Barclay YD, Antonellis KJ, Scherf U and Speed TP: Exploration, normalization and summaries of high density oligonucleotide array probe level data. Biostatistics 4: 249-264, 2003.

21. Smyth GK: Limma: Linear models for microarray data. Journal: 397-420, 2005.

22. Dennis G Jr, Sherman BT, Hosack DA, Yang J, Gao W, Lane HC and Lempicki RA: DAVID: Database for annotation, visualization and integrated discovery. Genome Biol 4: P3, 2003.

23. Ashburner M, Ball CA, Blake JA, Botstein D, Butler H, Cherry JM, Davis AP, Dolinski K, Dwight SS, Eppig JT, et al: Gene ontology: Tool for the unification of biology. The gene ontology. Nat Genet 25: 25-29, 2000.

24. Kanehisa M and Goto S: KEGG: Kyoto encyclopedia of genes and genomes. Nucleic Acids Res 28: 27-30, 2000.

25. Franceschini A, Szklarczyk D, Frankild S, Kuhn M, Simonovic M, Roth A, Lin J, Minguez P, Bork P, von Mering $\mathrm{C}$ and Jensen LJ: STRING v9.1: Protein-protein interaction networks, with increased coverage and integration. Nucleic Acids Res 41 (Database Issue): D808-D815, 2013.

26. Thakar A, Gupta P,Ishibashi T, FinnR, Silva-Moreno B,UchiyamaS, Fukui K, Tomschik M, Ausio J and Zlatanova J: H2A.Z and H3.3 histone variants affect nucleosome structure: Biochemical and biophysical studies. Biochemistry 48: 10852-10857, 2009.

27. Backs J and Olson EN: Control of cardiac growth by histone acetylation/deacetylation. Circ Res 98: 15-24, 2006.

28. Montgomery RL, Davis CA, Potthoff MJ, Haberland M, Fielitz J, Qi X, Hill JA, Richardson JA and Olson EN: Histone deacetylases 1 and 2 redundantly regulate cardiac morphogenesis, growth and contractility. Genes Dev 21: 1790-1802, 2007.

29. Orphanides G and Reinberg D: A unified theory of gene expression. Cell 108: 439-451, 2002

30. Wyrick JJ, Holstege FC, Jennings EG, Causton HC, Shore D, Grunstein M, Lander ES and Young RA: Chromosomal landscape of nucleosome-dependent gene expression and silencing in yeast. Nature 402: 418-421, 1999.

31. Touvron M, Escoubet B, Mericskay M, Angelini A, Lamotte L, Santini MP, Rosenthal N, Daegelen D, Tuil D and Decaux JF: Locally expressed IGF1 propeptide improves mouse heart function in induced dilated cardiomyopathy by blocking myocardial fibrosis and SRF-dependent CTGF induction. Dis Model Mech 5: 481-491, 2012.

32. van Almen GC, Verhesen W, van Leeuwen RE, van de Vrie M, Eurlings C, Schellings MW, Swinnen M, Cleutjens JP, van Zandvoort MA, Heymans S and Schroen B: MicroRNA-18 and microRNA-19 regulate CTGF and TSP-1 expression in age-related heart failure. Aging Cell 10: 769-779, 2011

33. Lang C, Sauter M, Szalay G, Racchi G, Grassi G, Rainaldi G, Mercatanti A, Lang F, Kandolf R and Klingel K: Connective tissue growth factor: A crucial cytokine-mediating cardiac fibrosis in ongoing enterovirus myocarditis. J Mol Med (Berl) 86: 49-60, 2008.
34. Broglio F, Benso A, Arvat E, Aimaretti G, Gottero C, Granata R, Boghen MF, Bobbio M, Camanni F and Ghigo E: Normal IGF-I and enhanced IGFBP-3 response to very low rhGH dose in patients with dilated cardiomyopathy. J Endocrinol Invest 23: 520-525, 2000.

35. Pucci A, Zanini C, Granata R, Ghignone R, Iavarone A, Broglio F, Sorrentino P, Bergamasco L, Rinaldi M and Ghigo E: Myocardial insulin-like growth factor-1 and insulin-like growth factor binding protein-3 gene expression in failing hearts harvested from patients undergoing cardiac transplantation. J Heart Lung Transplant 28: 402-405, 2009.

36. Hassfeld S, Eichhorn C, Stehr K, Naegele H, Geier C, Steeg M, Ranke MB, Oezcelik C and Osterziel KJ: Insulin-like growth factor-binding proteins 2 and 3 are independent predictors of a poor prognosis in patients with dilated cardiomyopathy. Heart 93: 359-360, 2007.

37. Khodabukus A and Baar K: Glucose concentration and streptomycin alter in vitro muscle function and metabolism. J Cell Physiol 230: 1226-1234, 2015.

38. Van Linthout S,Seeland U, Riad A,Eckhardt O,Hohl M, DhayatN, Richter U, Fischer JW, Böhm M and Pauschinger M, et al: Reduced MMP-2 activity contributes to cardiac fibrosis in experimental diabetic cardiomyopathy. Basic Res Cardiol 103: 319-327, 2008

39. Lakdawala NK, Thune JJ, Colan SD, Cirino AL, Farrohi F Rivero J, McDonough B, Sparks E, Orav EJ, Seidman JG, et al: Subtle abnormalities in contractile function are an early manifestation of sarcomere mutations in dilated cardiomyopathy. Circ Cardiovasc Genet 5: 503-510, 2012

40. Parsons JT, Horwitz AR and Schwartz MA: Cell adhesion: Integrating cytoskeletal dynamics and cellular tension. Nat Rev Mol Cell Biol 11: 633-643, 2010.

41. Podewski EK, Hilfiker-Kleiner D, Hilfiker A, Morawietz H, Lichtenberg A, Wollert KC and Drexler H: Alterations in Janus kinase (JAK)-signal transducers and activators of transcription (STAT) signaling in patients with end-stage dilated cardiomyopathy. Circulation 107: 798-802, 2003.

42. Hilfiker-Kleiner D, Hilfiker A, Fuchs M, Kaminski K, Schaefer A, Schieffer B, Hillmer A, Schmiedl A, Ding Z, Podewski E, et al: Signal transducer and activator of transcription 3 is required for myocardial capillary growth, control of interstitial matrix deposition, and heart protection from ischemic injury. Circ Res 95: 187-195, 2004.

43. McMullen JR, Amirahmadi F, Woodcock EA, Schinke-Braun M, Bouwman RD, Hewitt KA, Mollica JP, Zhang L, Zhang Y, Shioi $\mathrm{T}$ et al: Protective effects of exercise and phosphoinositide 3-kinase $(\mathrm{p} 110 \alpha)$ signaling in dilated and hypertrophic cardiomyopathy. Proc Natl Acad Sci USA 104: 612-617, 2007.

44. Waardenberg AJ,Bernardo BC,Ng DC, ShepherdPR,Cemerlang N, Sbroggiò M, Wells CA, Dalrymple BP, Brancaccio M, Lin RC and McMullen JR: Phosphoinositide 3-kinase (PI3K (p110 $\alpha)$ ) directly regulates key components of the Z-disc and cardiac structure. J Biol Chem 286: 30837-30846, 2011.

45. Molina-Navarro MM, Roselló-Lletí E, Tarazón E, Ortega A, Sánchez-Izquierdo D, Lago F, González-Juanatey JR, García-Pavía P, Salvador A, Montero JA, et al: Heart failure entails significant changes in human nucleocytoplasmic transport gene expression. Int J Cardiol 168: 2837-2843, 2013.

46. Molina-Navarro MM, Triviño JC, Martínez-Dolz L, Lago F, González-Juanatey JR, Portolés M and Rivera M: Functional networks of nucleocytoplasmic transport-related genes differentiate ischemic and dilated cardiomyopathies. A new therapeutic opportunity. PLoS One 9: e104709, 2014.

47. Dickover M, Hegarty JM, Ly K, Lopez D, Yang H, Zhang R, Tedeschi N, Hsiai TK and Chi NC: The atypical Rho GTPase, RhoU, regulates cell-adhesion molecules during cardiac morphogenesis. Dev Biol 389: 182-191, 2014.

48. Zhang Y, Li W, Zhu M, Li Y, Xu Z and Zuo B: FHL3 differentially regulates the expression of $\mathrm{MyHC}$ isoforms through interactions with MyoD and pCREB. Cell Signal 28: 60-73, 2016.

49. Abraham WT, Gilbert EM, Lowes BD, Minobe WA, Larrabee P, Roden RL, Dutcher D, Sederberg J, Lindenfeld JA, Wolfel EE, et al: Coordinate changes in Myosin heavy chain isoform gene expression are selectively associated with alterations in dilated cardiomyopathy phenotype. Mol Med 8 . 750-760, 2002. 